\title{
Comparison of epidural and spinal analgesia for patients undergoing laparoscopic ventral rectopexy within fast-track
}

\section{care}

\author{
A. Ehrlich ${ }^{1}$, M. Kairaluoma ${ }^{1}$, B. Wagner ${ }^{2}$ and I. Kellokumpu ${ }^{1 *}$ \\ ${ }^{1}$ Department of Surgery, Central Hospital of Central Finland, Finland \\ ${ }^{2}$ Department of Anaesthesiology, Uppsala Academic University Hospital, Sweden
}

\begin{abstract}
Background: To compare the effects of epidural and spinal analgesic regimens on outcomes for patients undergoing laparoscopic ventral rectopexy (LVR) within a fast-track care pathway.

Material and methods: This study is a retrospective analysis of prospectively collected data from two time periods. From 2007 to 2009 , a total of 38 consecutive patients underwent a standardized LVR within fast-track care pathway and epidural analgesia. The spinal analgesia group consisted of 42 consecutive patients who received similar LVR from 2013 to 2014. Totally intravenous anesthesia regimen and fast-track care pathway were similar during both study periods. The main measures of outcome were postoperative hospital stay, pain scores, and postoperative opioid consumption with 30-day morbidity and readmission rates as secondary outcomes.
\end{abstract}

Results: The study groups were well balanced for baseline characteristics. Postoperative hospital stay was shorter in the spinal than in the epidural group (median 2 versus 3 days, p $<0.02$ ). Fifteen of the 42 patients (35.7\%) in the spinal group were discharged on postoperative day one versus none in the epidural group. Pain scores were higher in the epidural group on postoperative day one. There were no deaths and only one complication and one conversion to open surgery in the spinal group.

Conclusion: Spinal analgesia is a safe analgesic regimen for patients undergoing LVR, and improves mobilization and shortens postoperative stay compared with epidural analgesia in the fast-track setting.

\section{Introduction}

Pelvic organ prolapse is common occurring in up to $40 \%$ of parous women [1]. Laparoscopic ventral rectopexy (LVR) is increasingly used for the treatment of rectal prolapse and for symptomatic high-grade (Oxford Grade 3-4) internal rectal prolapse [2].

Randomized trials and meta-analyses have now demonstrated the safety and efficacy of fast-track perioperative care in colorectal surgery, not only in reducing postoperative hospital stay and morbidity, but also in improving patient convalescence and satisfaction when compared with traditional care [3-6]. The role of fast-track perioperative care after LVR is not well known [2].

Optimal analgesia is an essential component of the fast-track program as it would facilitate earlier mobilization and oral intake, thus leading to shorter length of hospital stay [4-6]. For patient undergoing laparoscopic colorectal surgery the main analgesic method used so far has been epidural analgesia, which has been adopted from open colorectal surgery [7-8]. A recent randomized trial, however, showed that many of the outcomes in the epidural analgesia groups were significantly worse than those in the spinal analgesia and patientcontrolled anesthesia (PCA) groups [9].

While rationalizing the perioperative care for colorectal surgery the aim of this study was to examine the impact of epidural versus spinal analgesic regimen on outcomes for patients undergoing standardized LVR within a fast-track care pathway.

\section{Methods}

\section{Patients and study design}

This study is a retrospective analysis of prospectively collected data from two time periods. From 2007 to 2009, a total of 38 consecutive patients underwent a standardized LVR within fast-track care pathway and epidural analgesia. The spinal analgesia group consisted of 42 consecutive patients who received similar LVR from 2013 to 2014. Totally intravenous anesthesia and similar fast-track care pathways were used during both study periods. The main measures of outcome were postoperative hospital stay, pain scores, and postoperative opioid consumption with 30 -day morbidity and readmission rates as secondary outcomes. The study was approved by the ethics committee of the Central Hospital of Central Finland.

Indicationsfor surgerywere completerectal prolapse or symptomatic internal rectal prolapse (Oxford grade III-IV) accompanied with large rectocele or enterocele and no response to conservative therapy. Patients having the American Society of Anesthesiologists (ASA) score

Correspondence to: Ilmo Kellokumpu, Department of Surgery, Central Hospital of Central Finland Keskussairaalantie 19, 40620 Jyväskylä, Finland, Fax: +35814-2692929; E-mail: ilmo.kellokumpu@ksshp.fi

Key words: spinal analgesia, fast track care, laparoscopic ventral rectopexy

Received: April 29, 2016; Accepted: May 27, 2016; Published: May 31, 2016 
I-III, a good mental and physical performance status and having an attending person at home were included. Elderly patients fulfilling the predefined criteria were also included.

Preoperatively all patients underwent a complete history and physical examination and dynamic fluoroscopic defaecography with vaginal contrast agent or MRI proctography to detect intussusception and other concomitant pelvic floor disorders. Colonoscopy was performed selectively when clinically indicated. All patients were followed up for 30 days to assess morbidity and readmissions. Data was collected from a prospectively maintained, institutionally approved and password protected electronic colorectal database.

\section{Surgical technique}

The operation was standardized LVR using the four-trocar technique, and performed by two senior staff surgeons with the participation of residents in training [10]. An autonomic nervesparing anterior rectal mobilization was performed to levator level. Polypropylene or polyester mesh was sutured distally to the both sides of levator muscles, and attached to the rectum with four to six interrupted sutures and to the vagina with tow stiches. The proximal end of mesh was anchored to the sacral promontory using non-absorbable tackers.

\section{Fast-track protocol}

In accordance with a recent international consensus review the fast-track protocol included 20 evidence-based fast-track elements $[11,12]$. Preoperatively patients received extensive counseling, oral carbohydrates until two hours before surgery, and no mechanical bowel preparation. All patients received antimicrobial and thrombosis prophylaxis. The intraoperative protocol during both study periods consisted of standardized totally intravenous anesthesia (TIVA) using short-acting anesthetics ((induction with Propofol 1.0-2.5 mg/kg, Remifentanil $0.5-1.5 \mu \mathrm{g} / \mathrm{kg}$ and Cis-atracurium $0.1-0.15 \mathrm{mg} / \mathrm{kg}$ as bolus iv injection, maintenance with Propofol $2-4 \mathrm{mg} / \mathrm{kg} / \mathrm{h}$ and Remifentanil $0.1-0.5 \mu \mathrm{g} / \mathrm{kg} / \mathrm{min}$ and bolus injections of Cis-atracurium $0.02-0.03$ $\mathrm{mg} / \mathrm{kg}$ for muscle relaxation), intraoperative epidural analgesia with Ropivacain $0.2 \% 18 \mathrm{ml}$ and Fentanyl $100 \mathrm{ug}$ as bolus $(5-10 \mathrm{ml}$ preoperatively, followed by $2-5 \mathrm{ml}$ every $60-90$ minutes), restrictive to moderate administration of crystalloid solutions intra-operatively and crystalloid solution postoperatively on the day of surgery until next morning.

In the epidural group postoperative opioid-sparing analgesia (ropivacaine $1.23-1.29 \mathrm{mg} / \mathrm{ml}$ and fentanyl $3.07-3.23 \mu \mathrm{g} / \mathrm{ml}$ ) with epidural catheter (level Th9-11) and a single-use pump was scheduled to be removed on the second postoperative day. Oral paracetamol was started on the first postoperative day and NSAID-medication 2 days after surgery. Opioids were used for breakthrough pain. Ondansetron for postoperative nausea was used, if necessary.

In the spinal group analgesia involved injection of intrathecal anaesthesthetic before induction of general anaesthesia. This was performed at the L2-3 or L3-4 interspace using a combination of bupivacaine $(5 \mathrm{mg})$, fentanyl (10-20 $\mu \mathrm{g})$ and morphine $(100-160 \mu \mathrm{g})$. All patients in the spinal group received preoperatively ondansetron 4 $\mathrm{mg}$ and betamethasone $4-8 \mathrm{mg}$ per orally.

Furthermore, fast-track recovery was protocol driven with discontinuation of iv-fluids as soon as possible, early oral intake of fluids and postoperative feeding, removal of urinary catheter on the first postoperative day and early mobilization. In both study groups patients were discharged when they were afebrile, fully mobilized, tolerated three meals per day, had adequate pain control on oral analgesics and adequate home support.

\section{Postoperative follow-up}

Patients were viewed 3-5 times daily by operating surgeons and staff nurses. Dysuria, dizziness, hypotonia, cessation of nausea, vomiting and tolerance of full diet was recorded. If patient was out of bed less than six hours per day, the aim of early mobilization was not fulfilled. Pain scores were recorded 3-5 times daily by a specific nurse for pain management using a ten point visual analogue scale and a mean daily pain score was calculated. Mean number of systemic daily opioid doses (one opioid dose is oral oxynorm 5-10 mg or intramuscular oxanest 8-10 mg) was calculated.

\section{Statistical analysis}

Results are given as mean (SD) or median (interquartile range, IQR). The comparison between groups was made using t-test, MannWhitney, Kruskall-Wallis or chi-square test. All statistical tests were two sided, $p<0,05$ considered as statistically significant. Statistical analysis were conducted using SPSS (version 22.0 for Window; SPPS Inc., Chicago, IL, United States) software.

\section{Results}

Baseline clinical and treatment characteristics did not differ significantly between the study groups (Table 1). Complete rectal prolapse and internal rectal prolapse were evenly distributed in the study groups. The median duration of epidural analgesia was 2 (IQR22) days.

Postoperative hospital stay was shorter in the spinal group than in the epidural group [median 2 (IQR 1-3) days versus 3 (IQR2-3) days $(\mathrm{p}<0.001)]$ (Table 1$)$. Fifteen of the 42 patients $(35.7 \%)$ patients in the

Table 1. Baseline clinical characteristics,intraoperative data and surgical outcome.

\begin{tabular}{|c|c|c|c|}
\hline & $\begin{array}{c}\text { Epidural } \\
\mathrm{N}=\mathbf{3 8}\end{array}$ & $\begin{array}{c}\text { Spinal } \\
\mathrm{N}=42\end{array}$ & P-value \\
\hline Age, mean (SD) & $61.4(15.2)$ & $64(14.3)$ & 0.42 \\
\hline Female sex, n (\%) & $38(100)$ & $40(95.2)$ & 0.2 \\
\hline BMI, mean (SD) & $26.1(4.1)$ & $25.7(3.5)$ & 0.45 \\
\hline ASA I-II, n (\%) & $31(73.8)$ & $28(66.7)$ & 0.5 \\
\hline Comorbidity, $\mathrm{n}$ of patients $(\%)^{a}$ & $17(44.7)$ & $25(59.5)$ & 0.45 \\
\hline Cardiac & 6 & 7 & \\
\hline Hypertension & 9 & 16 & \\
\hline Pulmonary & 4 & 3 & \\
\hline Diabetes & 1 & 1 & \\
\hline Hypothyreosis & 1 & 5 & \\
\hline Other & 4 & 9 & \\
\hline \multicolumn{4}{|l|}{ Diagnosis, $\mathrm{n}(\%)$} \\
\hline Rectal prolapse & 16 & 17 & \\
\hline Intussuseptio & 22 & 25 & \\
\hline Operation time, median(IQR) & $114(98.5-130.0)$ & $105(76.5-127.3)$ & 0.2 \\
\hline Bleeding, median(IQR) & $5(0.8-10)$ & $20(10-50)$ & $<0.001$ \\
\hline Conversion rate, $\mathrm{n}(\%)$ & 0 & $1(2.4)$ & - \\
\hline Mortality (30d), n(\%) & 0 & 0 & - \\
\hline LOS, median(IQR) & $3(2-3)$ & $2(1-3)$ & $<0.001$ \\
\hline Readmission, $\mathrm{n}(\%)$ & $1(2,6)$ & $4(9.5)$ & 0.36 \\
\hline
\end{tabular}

${ }^{a}$ Figures in the columns are not additive because some patients had more than one comorbid condition 
spinal group were discharged on postoperative day one versus none in the epidural group. Mean pain scores were similar in both study groups on the day of surgery but significantly higher in the epidural group on postoperative day one (Table 2). More than four pain score value was in 4 patients from epidural group and only in one patient from spinal group on postoperative day one. Mean systemic opioid consumption (one dose 5-10 mg) was higher in the spinal group than in the epidural group on postoperative day one. However, on postoperative day two after removal of epidural catheter the opioid consumption was higher in epidural group. Inadequate mobilization during first and second postoperative days was more frequently observed in the epidural group. Surgery and anesthesia-related side effects, such as postoperative nausea and vomiting (PONV), dizziness and hypotonia were infrequent in both study groups.

Conversion to open surgery occurred in one patient in spinal group due to severe adhesions from previous open surgeries. There were no deaths. One patient had a small presacral haematoma (Table 1). Readmission rates did not differ significantly between epidural or spinal study groups $(2.6 \%$ versus $9.5 \%, \mathrm{P}=0.36)$.

\section{Discussion}

Effective analgesia is a prerequisite to decrease surgical stress response and to enhance mobilization. For patient undergoing LVR the main anesthetic method used so far has been epidural anesthesia, which has been adopted from open colorectal surgery. This study indicates that spinal analgesia instead of epidural analgesia for LVR within fasttrack care is effective in reducing the length of hospital stay, pain scores on postoperative days one, and improving early mobilization.

The postoperative length of stay following LVR usually ranges from 3 to 6 days instead of 2 to 3 days in our study [13]. Fifteen of the 42 patients $(35.7 \%)$ patients in the spinal group were discharged on postoperative day one versus none in the epidural group. Powar et al. reported the efficacy and safety of same-day discharge after LVR in selected patients with pelvic floor disorder. In their study $67 \%$ of the patients were discharged on postoperative day one and early discharge was associated with younger age and private insurance status [14].

In our study spinal analgesia seemed to provide a good early pain relief during the first 24 hours but was associated with a higher mean systemic opioid consumption on postoperative day one compared to epidural analgesia. In contrast, postoperative opioid consumption was higher in the epidural group on day 2 when epidural catheter was removed. Multimodal analgesia avoiding routine use of systemic

Table 2. Postoperative data and pain scores during basic ward care.

\begin{tabular}{|l|c|c|c|}
\hline & $\begin{array}{c}\text { Epidural } \\
\mathbf{N = 3 8}\end{array}$ & $\begin{array}{c}\text { Spinal } \\
\mathbf{N = 4 2}\end{array}$ & P-value \\
\hline Mean pain score, mean VAS score (SD) & & & \\
\hline Day 0 & $0.68(1.05)$ & $0.74(1.15)$ & 0.66 \\
\hline Day 1 & $0.67(1.09)$ & $0.16(0.43)$ & $<0.01$ \\
\hline Opioid consumption, mean (SD) & & & \\
\hline Day 0 & $0.03(0.16)$ & $0.07(0.26)$ & 0.3 \\
\hline Day 1 & $0.26(1.03)$ & $0.93(1.50)$ & 0.03 \\
\hline Day 2 & $1.13(1.54)$ & $0.43(1.02)$ & 0.02 \\
\hline PONV, $n$ of patients (\%) & $6(15.8)$ & $6(14.3)$ & 0.9 \\
\hline Mobilized <6 hrs/day POD 1-2 & $11(28.9)$ & 0 & $<0.001$ \\
\hline Dysuria, n of patients (\%) & 0 & $2(4.8)$ & 0.2 \\
\hline Dizziness, n of patients (\%) & $1(2.6)$ & 0 & 0.3 \\
\hline Hypotonia $n$ of patients (\%) & $1(2.6)$ & 0 & 0.3 \\
\hline
\end{tabular}

POD postoperative day opioids is considered crucial within enhanced recovery pathways to reduce the use of opioids. Consequently oral paracetamol and NSAID were routinely used here in both study groups.

Continuous thoracic epidural analgesia has been considered beneficial in major open abdominal procedures to control pain and to decrease catabolism, paralytic ileus, nausea, and vomiting [11]. However, recent randomized studies reported no advantage of thoracic epidural analgesia over patient-controlled anesthesia and another study demonstrated a detrimental effect of epidural analgesia on recovery after laparoscopic colorectal surgery when compared with spinal analgesia or patient-controlled analgesia $[7,9,15,16]$. In our study single-dose spinal analgesia resulted in faster mobilization and seemed to provide a good early pain relief compared to epidural analgesia

The role of epidural analgesia in laparoscopic surgery is increasingly being questioned because of increased time to mobilization, higher hospital costs, and longer length of hospital stay and higher incidence of urinary track infections $[9,17]$. For open surgery, the recommendation is that the epidural should be left in situ for $48 \mathrm{~h}$. In our study the median duration of epidural analgesia was two days thereby potentially hindering the early discharge of patients. Of note, recently published guidelines from UK do no longer recommend epidural analgesia as standard therapy for pain control after laparoscopic colorectal surgery $[17,18]$.

Intrathecal analgesia with high dose morphine (10-20 mg) has been associated an analgesic effect lasting up to more than 48 hours. However, this may cause severe central neurologic and respiratory side effects [19]. Some authors have injected up to $0,3 \mathrm{mg}$ of intrathecal morfine in elderly patients undergoing open colorectal surgery and did not report either respiratory depression or excessive sedation [19]. Today, the intrathecal dosage of morphine rarely exceeds $0.3 \mathrm{mg}$, which provides a significant reduction of dangerous, respiratory depression, even in elderly patients [20]. In line with the recommendation of American Society of Anesthesiologists (ASA) [21] using low dose intrathecal morphine in our study no morphine-related respiratory depression or haemodynamic side effects were observed.

The transversus abdominal 1plane (TAP) bloc is a regional anesthesia technique that provides analgesia of the parietal peritoneum, the anterior abdominal wall and the skin, and has only recently been implemented in laparoscopic abdominal surgery [22]. This technique has been shown to reduce postoperative pain and to reduce short-term opioid use after laparoscopic colorectal surgery [22,23].

A major challenge with this study was the lack of randomization which may have caused some selection bias. Another limitation was that the research was conducted in one hospital. However, patient demographics, technique of laparoscopic ventral rectopexy, TIVAanesthesia, fast-track protocol, and discharge criteria were similar during the two study periods. Furthermoe, senior surgeons had a large experience in laparoscopic colorectal surgery, and the quality of surgery was good as reflected by the short-term surgical outcome. Currently there are several analgesic methods in colorectal surgery. The optimal pain pain management for patients undergoing LVR and laparoscopic colorectal surgery remains to be determined in future randomized clinical trials.

\section{Conclusions}

Spinal analgesia for LVR within fast-track perioperative care is safe, improves postoperative recovery and results in shorter hospital stay thereby improving the efficiency of fast-track perioperative care. 


\section{Acknowledgements}

The authors have no conflicts of interest to declare.

This study was approved by the ethics committee of the hospital.

\section{References}

1. Wu JM, Vaughan CP, Goode PS, Redden DT, Burgio KL, et al. (2014) Prevalence and trends of symptomatic pelvic floor disorders in U.S. women. Obstet Gynecol 123: 141-148. [Crossref]

2. Mercer-Jones MA, D'Hoore A, Dixon AR, Lehur P, Lindsey I, et al. (2014) Consensus on ventral rectopexy: report of a panel of experts. Colorectal Dis 16: 82-88. [Crossref]

3. Spanjersberg WR, Reurings J, Keus F, van Laarhoven CJ (2011) Fast track surgery versus conventional recovery strategies for colorectal surgery. Cochrane Database Syst Rev CD007635. [Crossref]

4. Li MZ, Xiao LB, Wu WH, Yang SB, Li SZ (2012) Meta-analysis of laparoscopic versus open colorectal surgery within fast-track perioperative care. Dis Colon Rectum 55: 821827. [Crossref]

5. Delaney CP, Zutshi M, Senagore AJ, Remzi FH, Hammel J, Fazio VW (2003) Prospective, randomized, controlled trial between a pathway of controlled rehabilitation with early ambulation and diet and traditional postoperative care after laparotomy and intestinal resection. Dis Colon Rectum 46: 851-859. [Crossref]

6. Anderson AD, McNaught CE, MacFie J, Tring I, Barker P, Michell CJ (2003) Randomized clinical trial of multimodal optimization and standard perioperative surgical care. Br J Surg 90: 1497-1504. [Crossref]

7. Zafar N, Davies R, Greenslade GL, Dixon AR (2010) The evolution of analgesia in an 'accelerated' recovery programme for resectional laparoscopic colorectal surgery with anastomosis. Colorectal Dis 12:119-124. [Crossref]

8. Senagore J, Delaney CP, Mekhail N, Dugan A, Fazio VW (2003) Randomized clinical trial comparing epidural anesthesia and patient-controlled analgesia after laparoscopic segmental colectomy. British Journal of Surgery 90: 1195-1199. [Crossref]

9. Levy BF, Scott MJ, Fawcett W, Fry C, Rockall TA (2011) Randomized clinical trial of epidural, spinal or patient-controlled analgesia for patients undergoing laparoscopic colorectal surgery. Br J Surg98: 1068-1078. [Crossref]

10. D’Hoore A, Cadoni R, Penninckx F (2004) Long-term outcome of laparoscopic ventral rectopexy for total rectal prolapse. Br J Surg 91: 1500-1505. [Crossref]

11. Lassen K, Soop M, Nygren J, Cox PB, Hendry PO, Spies C, et al. (2009) Consensus review of optimal perioperative care in colorectal surgery: Enhanced recovery after surgery (ERAS) group recommendations. Arch Surg144: 961-969. [Crossref]
12. Ehrlich A, Wagner B, Kairaluoma M, Mecklin J-P, Kautiainen H, Kellokumpu I (2014) Evaluation of a fast-track protocol for patients undergoing elective colorectal surgery. Scand J Surg 103: 182-188. [Crossref]

13. Formijne Jonkers HA, Poierrie N, Draaisma WA, Broeders IA, Consten EC (2013) Laparoscopic ventral rectopexy for rectal prolapse and symptomatic rectocele: an analysis of 245 consecutive patients. Colorectal Dis 15: 695-699. [Crossref]

14. Powar MP, Ogilvie JW Jr, Stevenson AR (2013) Day-case laparoscopic ventral rectopexy: an achievable reality. Colorectal Dis 15: 700-706. [Crossref]

15. Zutshi M, Delaney CP, Senagore AJ, Mekhail N Lewis B, Connor JT, Fazio VW (2005) Randomized controlled trial comparing the controlled rehabilitation with early ambulation and diet pathway vs the controlled rehabilitation with early ambulation and diet with preemptive epidural anesthesia/analgesia after laparotomy and intestinal resection. Am J Surg 189: 268-272. [Crossref]

16. Wongyingsinn M, Baldini G, Stein B, Charlebois P, Liberman S, Carli F (2012) Spinal analgesia for laparoscopic colonic resection using an enhanced recovery after surgery programme: better analgesia, but no benefits on postoperative recovery: a randomized controlled trial. Br J Anaesth 108: 850-856. [Crossref]

17. Joshi GP, Bonnet F, Kehlet H, PROSPECT collaboration (2013) Evidence-based postoperative pain management after laparoscopic colorectal surgery. Colorectal Dis 15: 146-155. [Crossref]

18. Gustafsson UO, Scott MJ, Schwenk W, Demartines N, Roulin D, Francis N, et al. (2013) Guidelines for perioperative care in elective colonic surgery: Enhanced Recovery After Surgery (ERAS(®)) Society recommendations. World J Surg 37: 259-284. [Crossref]

19. Bailey PL, Lu JK, Pace NL, Orr JA, White JL, et al. (2000) Effects of intrathecal morphine on the ventilatory response to hypoxia. N Engl J Med 343: 1228-1234. [Crossref]

20. Yamashita K, Fukusaki M, Ando Y Tanabe T, Terao Y, Sumikawa K (2009) Postoperative analgesia with minidose intrathecal morphine for bipolar hip prosthesis in extremely elderly patients. J Anesth. 23: 504-507. [Crossref]

21. American Society of Anesthesiologists Task Force (2009)Practice guidelines for the prevention, detection, and management of respiratory depression associated with neuraxial opioid administration. Anesthesiology 110: 218-230.

22. Niraj G, Kelkar A, Hart E, Horst C, Malik D, Yeow C, et. al. (2014) Comparison of analgesic efficacy of four-quadrant transversus abdominis plane (TAP) block and continuous posterior TAP analgesia with epidural analgesia in patients undergoing laparoscopic colorectal surgery: an open-label, randomized non-inferiority trial. Anaesthesia 69: 348-355. [Crossref]

23. Smith SR, Draganic B, Pockney P, Holz P, Holmes R, Mcmanus et al. (2015) Transversus abdominis plane blockade in laparoscopic colorectal surgery: a doubleblind randomized clinical trial. Int J Colorectal Dis 30: 1237-1245. [Crossref]

Copyright: $\odot 2016$ Ehrlich A. This is an open-access article distributed under the terms of the Creative Commons Attribution License, which permits unrestricted use, distribution, and reproduction in any medium, provided the original author and source are credited. 